\title{
Unified phase field model to simulate both intergranular and transgranular failure in polycrystalline aggregates
}

\author{
Soukaina Riad ${ }^{\mathrm{a}, \mathrm{b}}$, Didier Bardel ${ }^{\mathrm{b}}$, Julien Réthoréa ${ }^{\mathrm{a}, *}$ \\ ${ }^{a}$ Civil and Mechanical Engineering Research Institute (GeM), Centrale Nantes/ Université de \\ Nantes/CNRS, UMR 6183, 1 rue de la Nö̈, F-44321 Nantes, France \\ ${ }^{b}$ Framatome, 10 rue Juliette Récamier, 69456 Lyon cedex 06, France
}

\begin{abstract}
In this work we develop a new phase field method for modeling fracture in polycrystalline materials. Many studies addressed this problem by combining cohesive models at the grain boundaries and damage models for the bulk. In this paper, a unified formulation is proposed in the phase field framework. Anisotropic failure is considered within the grain. Each of the preferential failure direction is associated with a damage variable. An additional damage variable is dedicated to the grain boundaries which are considered of finite thickness. Furthermore the value of the fracture energy at the grain boundaries is set depending on the local misorientation. The obtained model allows modeling competition and interactions between intergranular and transgranular fracture. The proposed model is illustrated through several numerical examples including crack initiation and propagation in polycrystalline aggregates.
\end{abstract}

Keywords: Phase field, polycrystal, Crack propagation, Regularized interfaces, Intergranular failure, Transgranular failure

\section{Introduction}

Damage modeling is a discipline aimed at analyzing materials or structure's behavior under extreme loading and anticipating their degradation. Simulating damage at the microscopic level yields a more accurate prediction of material's strength and allows to understand the impact of microstructural features/defects on the material's lifetime. For

*julien.rethore@ec-nantes.fr 
polycrystalline materials, such as most of the ceramics, rocks and metals, the microstructural features, i.e, topology, crystal lattice orientation and grain boundaries characteristics have significant effects on physical and mechanical macroscopic properties. The two fracture modes observed in polycrystalline materials are intergranular, where the failure takes place along the grain boundaries, and transgranular, where the crack propagates through the grains. Modelling the evolution and competition/interactions between these two fracture modes is often needed, for example for environmental degradation (such as hydrogen embrittlement, stress corrosion cracking ... [1] [2]) or solar cell wafers [3, but requires powerful numerical tools.

Many numerical models have been developed to study fracture for polycrystalline materials ([4, [5], [6]). Recently, the phase field model has proven to be a powerful tool to simulate complex cracking phenomena $([7,[8])$. The phase field model is a versatile model using an internal length specially designed for modelling systems with sharp interfaces. The model consists in incorporating a continuous variable field $d$ allowing the distinction between the different physical phases of a system (fully broken or unbroken) with smooth transitions over a characteristic distance [9]. Phase field modelling is attractive because of its ability to simulate complex cracking processes including initiation, coalescence, propagation and bifurcation $([10,11], 12])$. Crack evolution is governed by an energy minimization problem proposed by Francfort and Marigo [13] for brittle failure in a finite element model with a fixed mesh: the free energy is composed of the elastic energy and the energy required to generate a free surface according to Griffith criterion. Several studies have been developed within the context of failure in anisotropic materials, [14], 15] and [16] incorporate higher order structural tensor to obtain the non-convex material fracture energy function and [17] introduce an equivalent surface energy density for the homogenized matrix and fiber materials.

In polycrystalline materials, the role of interfaces (grain boundaries) is of crucial importance for crack propagation modelling. The mechanical response of such systems is strongly dependent on the fundamental competition between the crack penetration into the bulk and the deflection along the interface. Nguyen et al. [18, [19]] proposed a phase field model for polycrystalline materials, where the interface failure is inspired by a cohesive law incorporated in the regularized variational framework. Interfaces are 
captured by a level set method. The model takes into account elastic anisotropy, surface energy anisotropy and failure in grain boundaries by using only phase field variables to simulate intergranular and transgranular fracture. Paggi et al. 20] proposed a similar phase field formulation to $([18,[19])$ but instead of using the level set method to capture the discontinuity at crack interfaces, they introduced interfaces using compatible finite element mesh. Hansen-Dorr et al. [[21, [22, [23, 24] have proposed the concept of diffuse interface to be incorporated in the bulk material using the phase field method. The regularized interface is defined as a narrow solid subdomain of a solid with a characteristic width and is assigned an interface fracture toughness which depends on the length scales of the interface, the crack, and on the fracture toughness of the surrounding bulk material. A compensation of this effect is proposed [23], 24]] by modifying the numerical interface fracture toughness.

The present contribution aims at developing a unified phase field formulation for polycrystalline materials, that is able to take into account both intergraular and transgranular fracture. Failure is considered as anisotropic within the grains and preferential cleavage directions are defined depending on the grain orientations. Each of these directions is associated with a phase field damage variable. An additional damage variable is dedicated to the grain boundaries. The fracture energy attributed to the surface energy density of this variable is considered as varying based on the distance to grain boundaries. This makes the grain boundaries interfaces of non-zero thickness. Furthermore, the value of the fracture energy at the grain boundaries is set depending on the local misorientation.

This paper is structured as follows: Section 2 introduces the phase field method, then the main aspects of the proposed modeling at the polycrystal scale are presented. For this last aspect, special attention is paid to the modeling of (i) transgranular anisotropic failure, (ii) branching and multi-cracking phenomena and (iii) the spatial variation of the fracture toughness to control transition between intergranular and transgranular failure. In section 3, several numerical examples are explored to illustrate and discuss the potential of the proposed model. 


\section{Methods}

\subsection{Review of the Phase Field Method}

\subsubsection{Regularized variational framework}

The crack geometry is approximated in a regularized framework by a damage field $d$ such that $d=1$ on the cracked area $\Gamma$ and $d=0$ away from it. Fig. 1 illustrates the crack topology regularisation where $l$ is the characteristic regularization length describing the actual thickness of the diffuse crack. The variational approach to fracture following 10 .

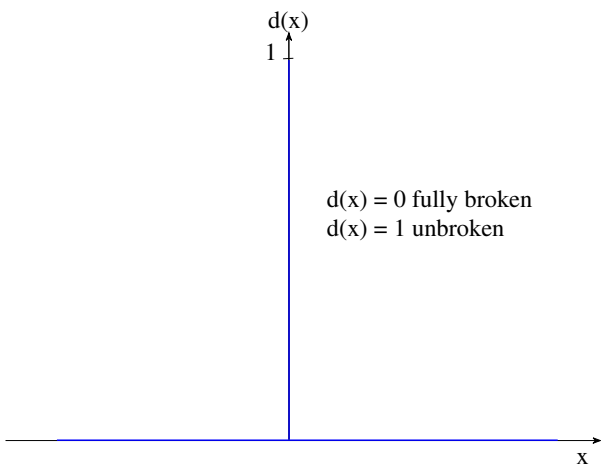

(a)

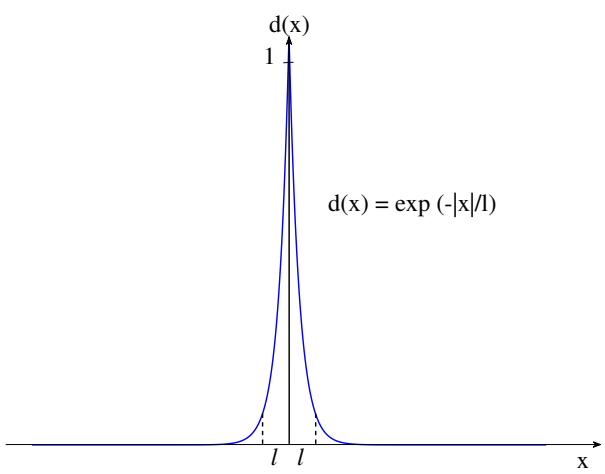

(b)

Figure 1: Crack topology approximation at $\mathrm{x}=0$ in a 1D case: (a) Sharp crack; (b) Diffusive crack with the length scale 1

defines the total energy for a cracked body as:

$$
E=E_{u}+E_{s}=\int_{\Omega} g(d) W_{u}(\varepsilon) d \Omega+G_{c} \int_{\Omega} \gamma(d, \nabla d) d \Omega
$$

where $E_{u}$ represents the elastic energy stored in the cracked body which can be expressed as the integral of the product of the strain energy density $W_{u}(\varepsilon)=\frac{1}{2} \varepsilon: \mathbb{C}: \varepsilon$ ( $\varepsilon$ is the strain tensor and $\mathbb{C}$ the elastic tensor of the material) and a degradation function describing the damage of the material as a function of the evolution of the phase field $d$ and is usually chosen to have the simple shape [25]:

$$
g(d)=(1-d)^{2}
$$


$E_{s}$ denotes the energy required to create/propagate the crack according to the Griffith criterion which is written as the product of the fracture energy $G_{c}$ (energy required to create a unit surface crack) and the crack surface functional. $W$ is the free energy:

$$
W=g(d) W_{u}(\varepsilon)+G_{c} \gamma(d) .
$$

where $\gamma(d, \nabla d)$ represents the crack density function per unit volume, defined by:

$$
\gamma(d, \nabla d)=\frac{1}{2 l} d^{2}+\frac{l}{2} \nabla d . \nabla d .
$$

The variational derivative of this function is: $\delta \gamma(d, \nabla d)=\frac{\partial \gamma}{\partial d}=\frac{d}{l}-l \Delta d$.

\subsubsection{Basics of thermodynamics and damage governing equation}

Assuming isothermal processes, the Clausius-Duhem inequality is expressed as:

$$
\sigma: \dot{\varepsilon}-\dot{W} \geq 0
$$

where $\sigma$ is the Cauchy stress. After development of Eq5, one obtains:

$$
-\frac{\partial W}{\partial d} \cdot \dot{d} \geq 0
$$

Since the damage process is irreversible $(\dot{d}>0)$, by substituting Eq. 3 in Eq. 6 , one obtains:

$$
2(1-d) W_{u}-\frac{G_{c}}{l}\left(d-l^{2} \Delta d\right)=0 \quad \text { if } \quad \dot{d}>0 .
$$

This evolution equation Eq. 7 governs the evolution law of the damage variable $d$. Therefore, Miehe et al. [12] introduced a strain history functional $\mathcal{H}$ to deal with the loading and unloading histories and to ensure the positivity of $\dot{d}$ even for unloading conditions:

$$
\mathcal{H}(\underline{x}, t)=\max _{\tau \in[0, t]}\left\{W_{u}(\underline{x}, \tau)\right\} .
$$

The phase field problem to be solved to evaluate the field $d(\underline{x}, t)$ at time $t$ is:

$$
\left\{\begin{array}{l}
2(1-d) \mathcal{H}-\frac{G_{c}}{l}\left(d-l^{2} \Delta d\right)=0 \quad \text { in } \quad \Omega, \\
d(\underline{x})=1 \quad \text { on } \quad \Gamma \\
\nabla d(\underline{x}) \cdot \underline{n}=0 \quad \text { on } \quad \partial \Omega .
\end{array}\right.
$$




\subsubsection{Hybrid formulation with spheric strain decomposition}

To avoid the issue of crack interpenetration under compression loading, an asymmetric damage formulation is used. The hybrid formulation proposed by Ambati et al. [26] is adopted in this work. This formulation keeps linear the displacement problem and the damage field evolution is only controlled by the part of elastic energy responsible of crack propagation $W_{u}^{+}$. To define the positive and negative part of the strain energy, the decomposition proposed by Amor et al. [27] is chosen since it is possible to apply it for anisotropic materials. The elastic strain is decomposed into a volumetric and a deviatoric part. Then, the damage is assumed to be driven by expansion (positive spherical part) and shear. Thus, depending on the sign of the spherical strain $(\operatorname{tr}(\varepsilon))$, the positive part (promoting failure) and negative part (not promoting failure) are defined as follows:

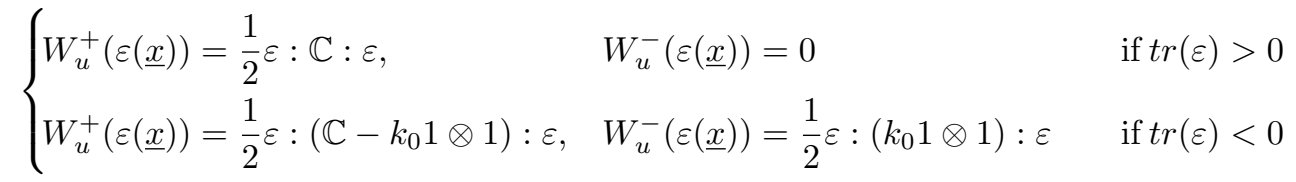

where $k_{0}$ is the bulk modulus for the undamaged material (relating the spherical part of the strain to the spherical part of the stress).

In the framework of the hybrid decomposition, the phase field problem to be solved is then:

$$
\left\{\begin{array}{l}
\mathcal{H}(\underline{x}, t)=\max _{\tau \in[0, t]}\left\{W_{u}^{+}(\varepsilon(\underline{x}, \tau))\right\} \\
2(1-d) \mathcal{H}-\frac{G_{c}}{l}\left(d-l^{2} \Delta d\right)=0
\end{array}\right.
$$

and the displacement problem is:

$$
\left\{\begin{array}{l}
\forall x: \quad \text { if } \quad W_{u}^{+}<W_{u}^{-} \text {then } \quad d=0 \\
\sigma=\frac{\partial W(\varepsilon, d)}{\partial \varepsilon}=(1-d)^{2} \frac{\partial W_{u}(\varepsilon)}{\partial \varepsilon}
\end{array}\right.
$$

In practice, a small parameter $k$ is introduced in Eq $12 \mathrm{~b}$ chosen as small as possible to maintain the well-posedness of the problem such as:

$$
\sigma=\frac{\partial W(\varepsilon, d)}{\partial \varepsilon}=\left((1-k)(1-d)^{2}+k\right) \frac{\partial W_{u}(\varepsilon)}{\partial \varepsilon}
$$


The constraint defined by Eq $12 \mathrm{a}$ is introduced in the hybrid formulation to prevent crack faces interpenetration. It comes with the governing equations Eq11b and Eq12b.

\subsection{Phase field method at the polycrystal scale}

In this section, we will focus on describing the phase field formalism at the polycrystal scale. To do so, we will describe how to introduce preferential crack propagation directions and how to produce multi-cracking. Then, we will consider the heterogeneous mechanical behaviour of the polycrystal to promote intergranular and/or transgranular cracks.

\subsubsection{Directionality effect}

For anisotropic crystals, crack propagation can take place along preferential planes (so-called cleavage planes). To model this directionality effect, we introduce in the expression of the cracked surface density Eq 4 a term that prevents damage increase along orientations normal to the cleavage plane :

$$
\gamma(d, \nabla d, \omega)=\frac{1}{2 l} d^{2}+\frac{l}{2} \omega:(\nabla d \otimes \nabla d)
$$

where $\omega$ is a second order tensor characterizing the failure anisotropy of the material (directionality). In order to make the energy release rate dependent on crack orientation, this term was defined by Clayton and Knap [28] as:

$$
\omega=1+\beta(1-\underline{M} \otimes \underline{M})
$$

where $\underline{M}$ is the unit vector normal to the preferential cleavage plane and $\beta$ is a factor used to penalize damage on the direction defined by $\underline{M}$. The variational derivative of the anisotropic crack surface density function $\gamma(d, \nabla d, \omega)$ is now defined as [29:

$$
\begin{aligned}
\delta \gamma(d, \nabla d, \omega) & =\frac{\partial \gamma}{\partial d} \\
& =\frac{d}{l}-l(\Delta d+\beta(\Delta d-\underline{M} \otimes \underline{M}: \nabla \otimes \nabla d)) .
\end{aligned}
$$

And the phase field problem to be solved at each time increment becomes: 


$$
\left\{\begin{array}{l}
2(1-d) \mathcal{H}-\frac{G_{c}}{l}\left(d-l^{2}(\Delta d+\beta(\Delta d-\underline{M} \otimes \underline{M}: \nabla \otimes \nabla d))\right)=0 \quad \text { on } \Omega, \\
d(\underline{x})=1 \quad \text { in } \quad \Gamma, \\
\nabla d(\underline{x}) \cdot \underline{n}=0 \quad \text { in } \quad \partial \Omega .
\end{array}\right.
$$

In order to analyze the modification introduced by the directionality formalism, we compute the tensor $\omega$ in Eq17a for a simple 2D example. We assume a material having a preferential direction $[1 ; 0]$, so $M=[0 ; 1]$. We obtain :

$$
2(1-d) \mathcal{H}-\left(\frac{G_{c}}{l} d-G_{c} l\left[\begin{array}{cc}
1+\beta & 0 \\
0 & 1
\end{array}\right]: \nabla \otimes \nabla d\right)=0 \quad \text { on } \Omega .
$$

One may notice that the fracture energy $G_{c}$ is modified in the preferential direction of propagation by the factor $1+\beta$. Thus, the directionality formalism increases the material fracture energy in the related cleavage plane. To prevent this increase, we propose a numerical correction on the fracture energy and on the internal length $l$ factors :

$$
G_{c c}=\frac{G_{c}}{\sqrt[4]{1+\beta}}, \quad l_{c}=\frac{l}{\sqrt[4]{1+\beta}} .
$$

This correction is chosen so as to keep the ratio $\frac{G_{c c}}{l_{c}}=\frac{G_{c}}{l}$ unchanged and to keep the value of the effective $G_{c}$ to its physical value in the cleavage plane. The numerical example in Section 3 demonstrate that this correction is efficient.

\subsubsection{Multi-cracking/crack branching}

Depending on their class of symmetry, crystals have multiple cleavage planes. In order to take into account this phenomenon, multiple phase fields $d_{i}$ are introduced to quantify the damage accumulation on each cleavage plane. Thus, the total cracked surface will be expressed as a summation of the multiple cracking surfaces by the following equation:

$$
\Gamma_{l}\left(d_{i}, w_{i}\right)=\sum_{i} \Gamma_{l}^{i}\left(d_{i}, w_{i}\right)=\sum_{i} \int_{\Omega} \gamma_{i}\left(d_{i}, \nabla d_{i}, \omega_{i}\right) d \Omega
$$

Then, Eq 3 Eq2 and Eq17a must be modified for the multi-phase field formalism. The total free energy is here rewritten as:

$$
W=W_{u}\left(\varepsilon(\underline{u}), d_{i}\right)+G_{c} \sum_{i} \gamma_{i}\left(d_{i}, \nabla d_{i}, \omega_{i}\right) .
$$


The elastic energy stored in the cracked body is affected by the damage accumulated on each cleavage plane. Then, Eq2 2 becomes:

$$
g\left(d_{i}\right)=\prod_{i}\left(1-d_{i}\right)^{2} .
$$

By applying the Clausius Duhem inequality for each orientation, we obtain the following governing equation for each phase field $d_{i}$ :

$$
2\left(1-d_{i}\right) \prod_{j \neq i}\left(1-d_{j}\right)^{2} W_{u}^{+}-G_{c} \delta \gamma(d, \nabla d, \omega)=0
$$

and strain history function associated to each phase field is defined as follows:

$$
\mathcal{H}_{i}(\underline{x}, t)=\max _{\tau \in[0, t]}\left\{\prod_{j \neq i}\left(1-d_{j}\right)^{2} W_{u}^{+}(\underline{x}, \tau)\right\} .
$$

The resulting system to be solved for each phase field $d_{i}$ is:

$$
\left\{\begin{array}{l}
\mathcal{H}_{i}(\underline{x}, t)=\max _{\tau \in[0, t]}\left\{\prod_{j \neq i}\left(1-d_{j}\right)^{2} W_{u}^{+}(\underline{x}, \tau)\right\} \\
2\left(1-d_{i}\right) \mathcal{H}_{i}-\frac{G_{c}}{l}\left(d_{i}-l^{2}\left(\Delta d_{i}+\beta_{i}\left(\Delta d_{i}-\underline{M}_{i} \otimes \underline{M}_{i}: \nabla \otimes \nabla d_{i}\right)\right)\right)=0 \quad \text { on } \quad \Omega
\end{array}\right.
$$

And the global displacement problem is:

$$
\left\{\begin{array}{l}
\forall x, \quad \forall i,: \quad W_{u}^{+}<W_{u}^{-} \quad \Rightarrow d_{i}=0 \\
\sigma=\frac{\partial W(\varepsilon, d)}{\partial \varepsilon}=\left((1-k) g\left(d_{i}\right)+k\right) \frac{\partial W_{u}(\varepsilon)}{\partial \varepsilon}
\end{array}\right.
$$

\subsubsection{Intergranular and transgranular failure}

The directionality formalism makes it possible to model transgranular failure but the model is also required to include intergranular failure as often encountered in physical applications. For this purpose, it is necessary to introduce a heterogeneous behaviour in the polycrystal to take into account the specific properties of the microstructure (especially the grain boundary zone). Therefore, the proposed model, based on the Griffith criterion, spatially distributes the $G_{c}$ parameter. Grain boundaries will be affected by lower $G_{c}$ values compared to the grain bulk and an additional damage variable will be dedicated to the grain boundaries. This phase field formulation is powerful since it dispenses with 
the cohesive zone models (CZM) classically adopted in the literature to simulate grain boundaries ([18, [19, [3]) and integrates all the parameters in a consistent and unified phase field formalism. Grain boundaries are thus considered as an interface of thickness $e_{p}$ larger than the internal length parameter associated with the grain boundary surface energy. The fracture energy distribution in the vicinity of these interfaces is based on the distance to grain boundaries.

Following a calculation of the distance from the material points to the adjacent grain boundaries $\Psi$, it is possible to prescribe a variation of the fracture energy for the intergranular damage variable $G_{c v}$ between $G_{c}^{B}$ within the grain and $G_{c}^{G B}$ along the grain boundaries. We carried out a study to chose the most appropriate function (see Appendix A and we finally chose a sinusoidal function as shown in Fig 2

$$
\begin{aligned}
G_{c v}= & \frac{G_{c}^{B}+G_{c}^{G B}}{2}+ \\
& \frac{G_{c}^{B}-G_{c}^{G B}}{2} \sin \left(\min \left(\frac{\pi}{2}, \frac{-\pi}{2}+\pi\left(\frac{|\Psi|}{e_{p}^{*}}\right)^{3}\right)\right) .
\end{aligned}
$$

$G_{c}^{B}$ is a numerical parameter, chosen as high as possible to activate the variable damage dedicated to the grain boundaries only for intergranular fracture. For the other damage variables, the fracture energy is set equal to its physical value $G_{c}$. $e_{p}^{*}$ is a fictitious thickness chosen so as to impose a $G_{c}$ value on the edge of the grain boundaries defined by its thickness $e_{p}: G_{c v}\left(e_{p}\right)=G_{c}$.

This sinusoidal function Eq. 27 provides a smooth transition between $G_{c}^{G B}$ and $G_{c}$ allowing to capture the grain boundaries area without requiring a numerically expensive mesh. However, as it was mentioned in $([23], 24])$, the actual value of the fracture energy along the grain boundaries depends on the ratios $G_{c}^{B} / G_{c}^{G B}, e_{p} / l$ and the chosen variation function. When the last ratio, $e_{p} / l$, becomes small, the dissipated energy is no more controled by $G_{c}^{G B}$ alone, but is also influenced by $G_{c}^{B}$. A numerical compensation for the sinusoidal function is proposed in Appendix B.

\subsubsection{Grain boundary misorientataion}

A polycrystal is composed of grains and grain boundaries where the fracture energy is lower than within the grains. Moreover, the formation history of each grain boundary is different, which can induce heterogeneities of the mechanical properties between grain 


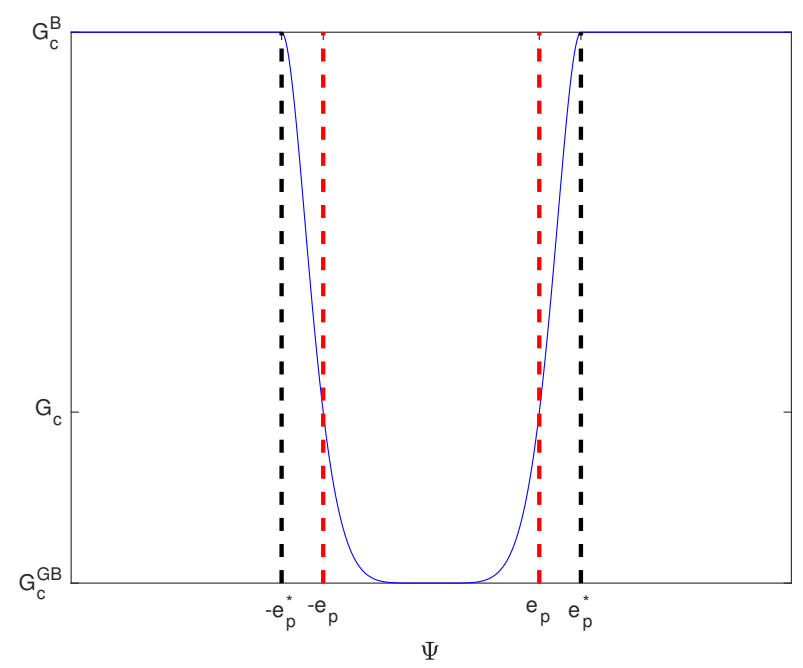

Figure 2: Fracture energy variation for the chosen sinusoidal function with the fictitious thickness

boundaries. As an example, this heterogeneity can be taken into account through the effect of grain boundaries misorientation which is calculated from the misorientation matrix H:

$$
H=R_{G 2} \cdot R_{G 1}^{T} .
$$

$R_{G 1}$ and $R_{G 2}$ are the rotation matrices of the two involved grains represented by Euler angle triplet of the local coordinates system of each grain with respect to the global system. The global misorientation angle (scalar parameter) is then obtained by the expression [30]:

$$
\theta=\operatorname{Arcos}\left(\frac{H_{11}+H_{22}+H_{33}-1}{2}\right) \text {. }
$$

Several formulations are able to take into account the effect of the misorientation angle on mechanical properties. In this paper, we consider a geometrical formulation used in the work [31:

$$
G_{c}^{G B M}=G_{c}^{G B^{a v g}}+\Delta G_{c}^{G B} \cos (4 \theta)
$$

where $G_{c}^{G B M}$ is the grain boundary fracture energy taking into account misorientation effects, $G_{c}^{G B^{a v g}}$ the average value of the grain boundary fracture energy deviation and $\Delta G_{c}^{G B}$ the maximal fracture energy deviation. Fig 3 shows that this function yields a periodic variation of the $G_{c}^{G B}$ with the misorientation angle that is consistent with the 


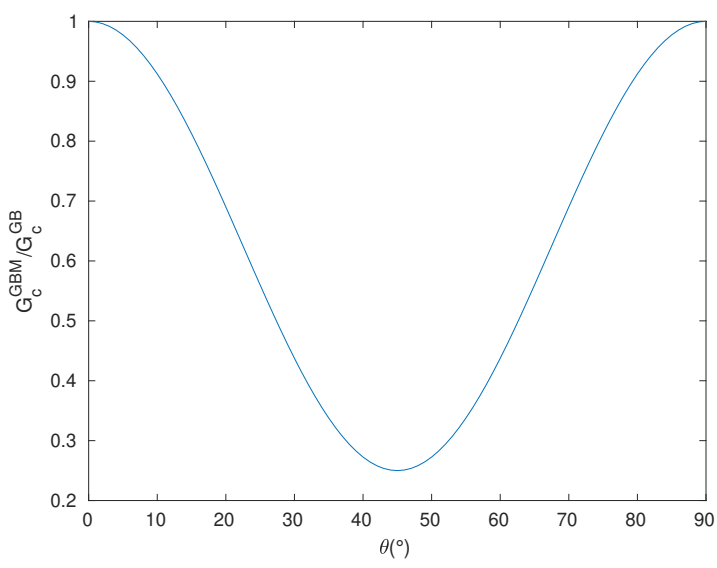

(a)

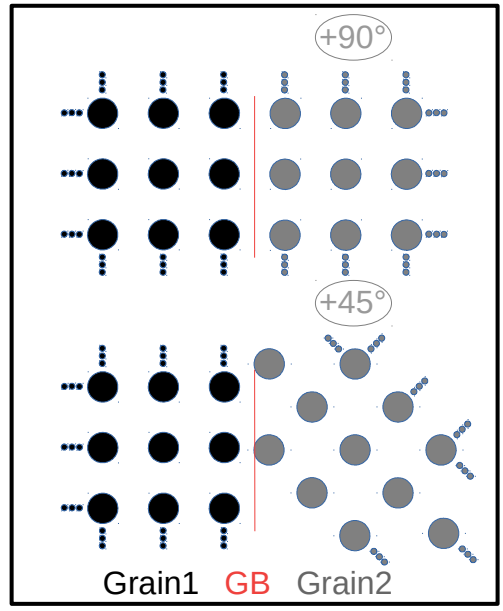

(b)

Figure 3: The effect of the misorientation angle on the grain boundary properties for a 2D problem: (a) fracture energy variation as a function of misorientation, (b) illustration of the effect of grains rotations on the relative positioning of the atoms on both sides of the grain boundary.

symmetry class of cubic lattice.

\subsection{Overall algorithm}

For a multi-phase field problem that can take into account inter and transgranular fractures, the algorithm for solving the displacement field and the phase fields problems is :

- Initialize the strain history function $\mathcal{H}_{i}^{0}$.

- For each phase field:

- Choose of the nature of $G_{c}$ spatial variation: variable for the phase field associated to grain boundaries Eq. 27 or constant for the other phase fields.

- Assign the unit vector normal to the preferential cleavage plane $\underline{M}$ and the associated $\beta$ coefficient for each transgranular failure phase field.

- For all loading increments (pseudo time $\left.t^{n+1}\right), \mathcal{H}^{n}$ is known:

1. For each damage field, compute $d_{i}^{n+1}$ by solving the linear phase field problem Eq. $25 \mathrm{~b}$. 
2. Compute the damage function $g\left(d_{i}\right)$ by Eq. 22 .

3. Compute $\underline{u}^{n+1}$ by solving the displacement problem Eq. $26 \mathrm{~b}$.

4. For each damage field, compute $\mathcal{H}_{i}^{n+1}$ the strain history function Eq. 25a.

5. $(.)^{n}$ receives $(.)^{n+1}$ and go to $(1)$.

\section{Numerical examples}

In this section, we will study some numerical examples to validate our proposals presented in the previous section. We will start by a test to validate the proposed correction of the directionality formalism. Then, we will present a benchmark problem to assess the new phase field model we propose.

In all examples the numerical parameters are summarized, then the results are shown and interpreted. All cases are in 2D under plane strain assumption. According to the results of Miehe et al. [12, the length scale parameter $l$ should always be taken at least two times larger than the smallest element size.

All simulations are performed in the finite element code Cast3M [32] where we have implemented the proposed phase field method in two dimensions.

\subsection{Directionality correction}

In this first example, we present a classical benchmark problem that is the single-edge notched tension test consisting in a square containing a straight horizontal notch. The geometry and the boundary conditions are shown in Fig 4 . The material parameters are the same as in Miehe et al.[12] and Ambati et al.[26] :

$$
\begin{aligned}
& \lambda=121.15 G P a, \quad \mu=80.77 G P a, \\
& G_{c}=2700 \mathrm{~J} / \mathrm{m}^{2}, \quad l=4 \times 10^{-6} \mathrm{~m}
\end{aligned}
$$

The spatial discretization of the model contains 27324 triangular elements, with an $a$ priori refined mesh where the crack propagation is expected. The displacement of the top edge is controled by increments: $\Delta u=1 \times 10^{-5} \mathrm{~mm}$ up to $u=5 \times 10^{-3} \mathrm{~mm}$ and then $\Delta u=1 \times 10^{-6} \mathrm{~mm}$ up to failure.

The result of this classical problem is the crack propagation in the horizontal direction as shown in Fig. 5. To assess the impact of directionality formalism, two simulations are 


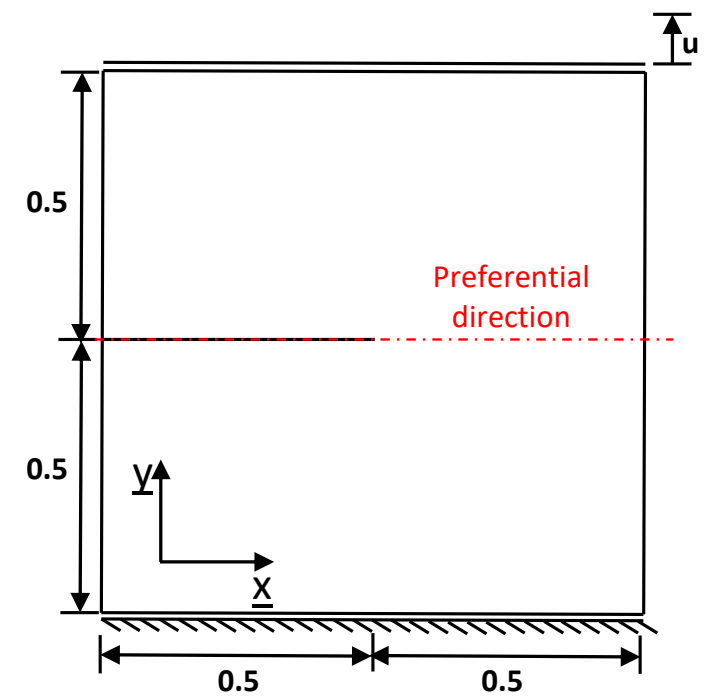

Figure 4: Geometry and boundary conditions of single-edge notched Tension test 
performed using the directionality formalism (by imposing $\underline{M}=[0 ; 1]$ and $\beta=20$ ) with and without the correction proposed in paragraph 2.2.1. Fig 6 shows comparison of loaddisplacement curves where it is clearly observed that the classical directionality formalism increases the material toughness (the red curve) since the force required to damage the material is greater than that required for the simulation without the directionality formalism (the blue curve). However, the corrected directionality formalism maintains the material toughness by yielding an almost identical load-displacement behavior (the black curve). One can notice that the amount of energy dissipated using the directionality corrected formalism is slightly lower than for the initial formalism. Indeed, as it is illustrated in (see Fig 5), the directionality formalism prevents damage to grow in the direction defined by $\underline{M}$ and the damage profile along this direction is sharper than using no directionality constraint. This is consistent with the above mentioned observation made from Fig 6 stating that the dissipation with directionality constraint is lower.

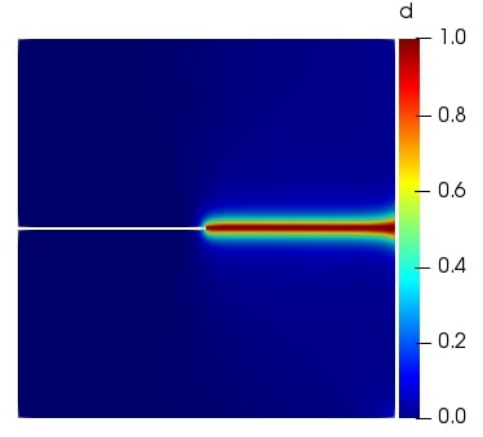

(a)

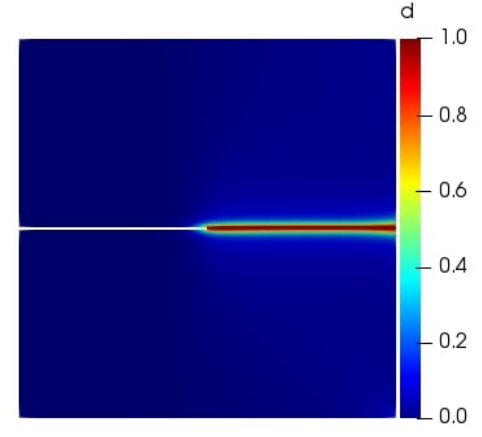

(b)

Figure 5: Crack phase-field for single-edge notched tension test for: (a) $\beta=0$ and (b) $\beta=20$.

This test allowed us to conclude on the necessity of correcting the increase in fracture energy due to the directionality formalism. Our proposed numerical correction seems to give promising results ensuring the mechanical fracture properties of the model material. 


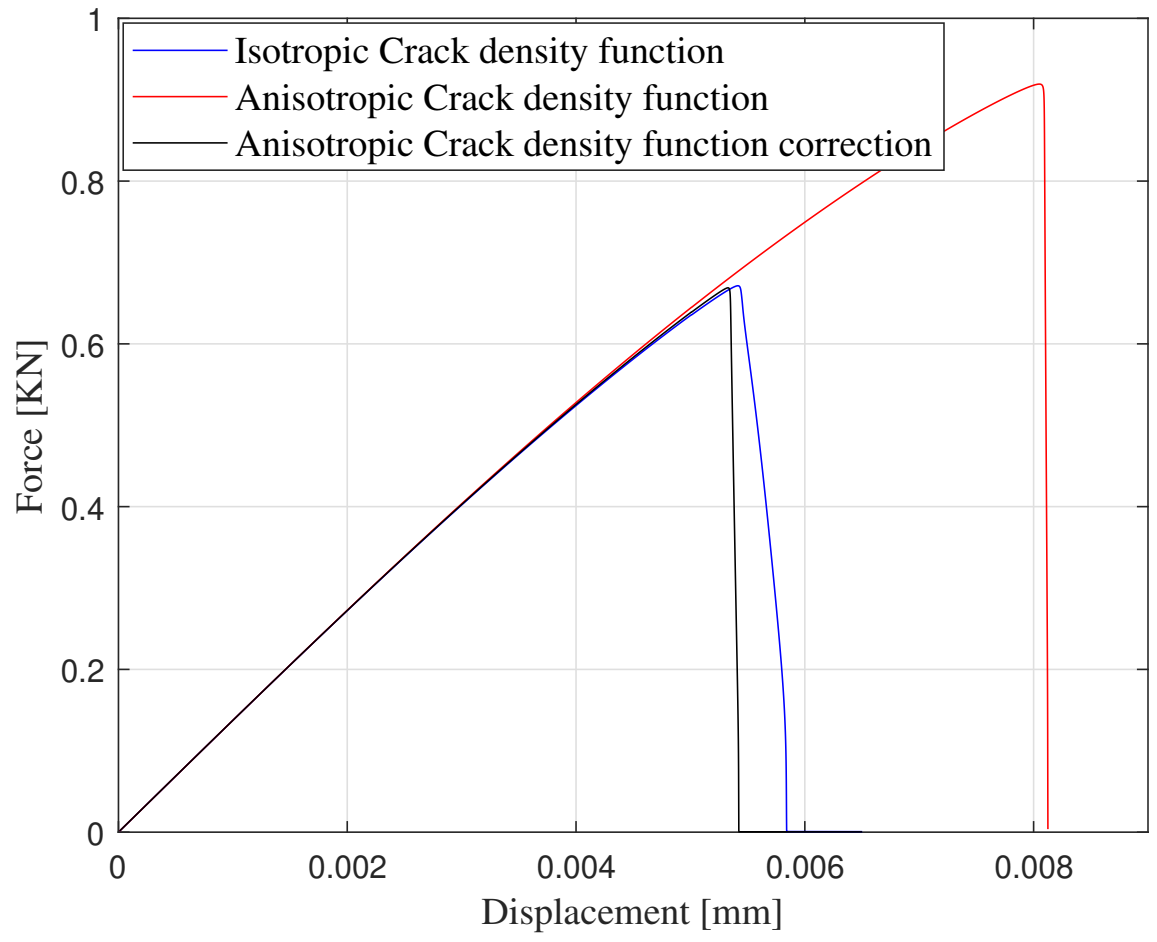

Figure 6: Comparison of the Load-displacement curves for the three studied cases 


\subsection{Intergranular and transgranular failure}

In this example, we will assess the potential of our phase field formulation to simulate both intergranular and transgranular fracture. To do this, the open source software Neper [33] was used to generate the geometry (5 grains) and the mesh (containing 78368 linear triangular elements). An initial crack is then introduced. Fig 7 a provides details about the geometry, boundary conditions and the orientation of each grain. The stiffness tensor of the material is considered of cubic symmetry in the local coordinates system such as:

$$
\mathbb{C}=\left[\begin{array}{ccc}
280 & 120 & 0 \\
120 & 280 & 0 \\
0 & 0 & 140
\end{array}\right] G P a
$$

The bulk modulus is $k_{0}=\left(C_{11}+2 C_{12}\right) / 3=173.3 \mathrm{GPa}$.

The fracture energy in the grains is set to $G_{c}=1500 \mathrm{~J} / \mathrm{m}^{2}$. The regularization parameters are set to $l=0.012 \mathrm{~mm}$ for the phase field and $e_{p}=0.015 \mathrm{~mm}$ for the grain boundaries thickness. The numerical parameters for fracture energy variation are set to $G_{c}^{B}=10 \times G_{c}$ and $e_{p}^{*}$ is calculated by the following equation:

$$
e_{p}^{*}=e p\left[\frac{1}{\pi}\left(A \sin \left(\frac{2 G_{c}-G_{c}^{B}-G_{c}^{G B}}{G_{c}^{B}-G_{c}^{G B}}\right)+\frac{\pi}{2}\right)\right]^{-\frac{1}{3}}
$$

The preferential cleavage plane is oriented at $0^{\circ}$ with respect to the local $x$-axis:

$$
M=[0 ; 1] \text { and } \beta=50
$$

The influence of the grain boundaries fracture energy $G_{c}^{G B}$ is analyzed by considering three different sets of parameters (the first case is represented in Fig $7 \mathrm{~b}$ ): (1) $G_{c}^{G B}=$ $0.8 G_{c} ;(2) G_{c}^{G B}=0.6 G_{c} ;(3) G_{c}^{G B}=0.3 G_{c}$. The grain boundaries fracture energy to be applied in the simulations are obtained by the compensation procedure Fig.B.2

In the first two cases, the fracture energy along the grain boundaries is chosen to promote transgranular fracture while the configuration $G_{c}^{G B}=0.3 G_{c}$ promotes intergranular fracture. The damage variable associated to the grain boundaries is denoted $d_{1}$ and that corresponding to the preferential direction $0^{\circ}$ is denoted $d_{2}$. We plot an equivalent damage field $d_{e q}=d_{1}+d_{2}-d_{1} d_{2}$ to visualize the final crack state. The computation is performed over 80 load increments of $u=10^{-4} \mathrm{~mm}$. The displacements 


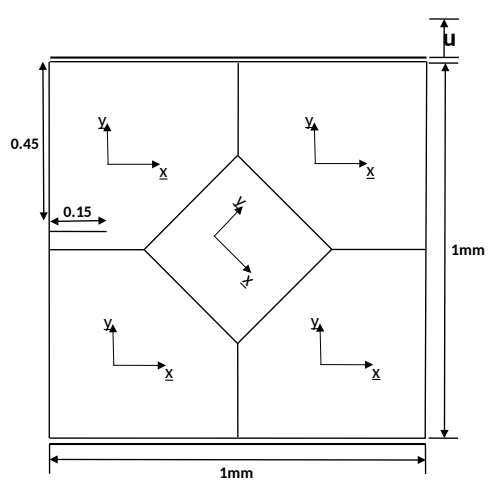

(a)

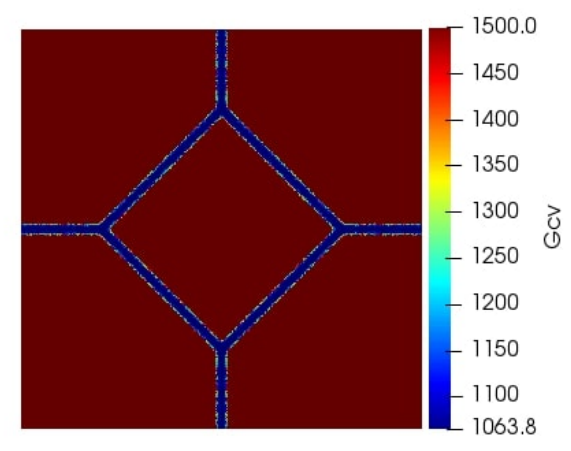

(b)

Figure 7: (a) Geometry, boundary conditions and grain direction for the polycrystalline structure containing 5 grains, (b) The element-wise field of fracture energy spatial variation for the first case: $G_{c}^{G B}=0.8 G_{c}$.

are prescribed along the $y$-direction for upper edge while the displacements along $x$ are free.

The results of the crack propagation for the three cases are depicted in Fig 8 , For the first case (Fig $8 \mathrm{a}$, the grain boundary fracture energy $G_{c}^{G B}$ is close to the one within the grains, then the fracture is trangranular. The crack propagates in the preferred direction and changes its orientation depending on the orientation of each grain. In the second case (Fig 8b), when the grain boundary fracture energy is 0.6 the grains one, the fracture is also transgranular but the crack has a tendency to run within the grain boundary at "the exit" of the central grain (but the energy was not yet sufficient to pursue this path). In the last case (Fig $8 \mathrm{c}$ ), the fracture is intergranular, the grain boundary fracture energy in this zone is small and the crack runs along until failure. These results demonstrate the ability of the proposed model to simulate fracture in polycrystalline materials.

\subsection{Grain boundary misorientation}

In order to investigate the influence of grain boundary misorientation on the fracture behavior, we consider case (2) of the previous problem, $G_{c}^{G B}=0.6 G_{c}$. In this simulation, the grain boundaries fracture energy is made dependent on grains misorientation as 

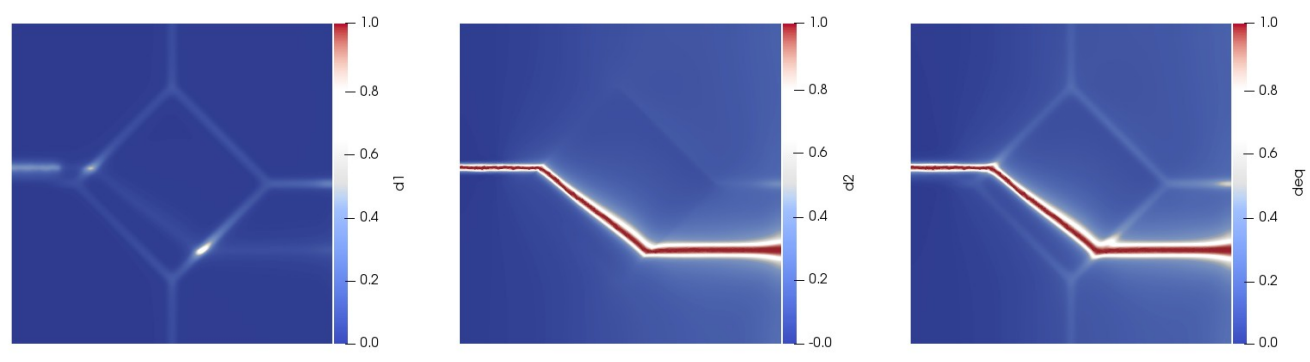

(a)
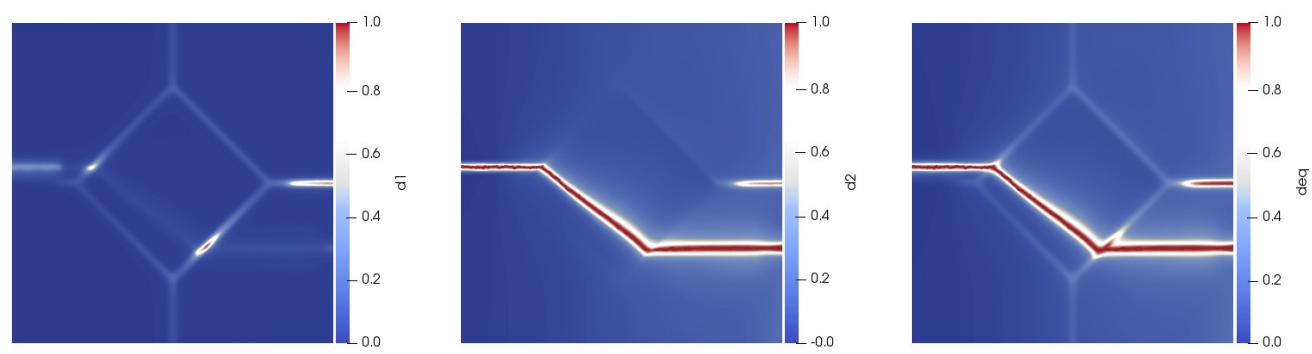

(b)
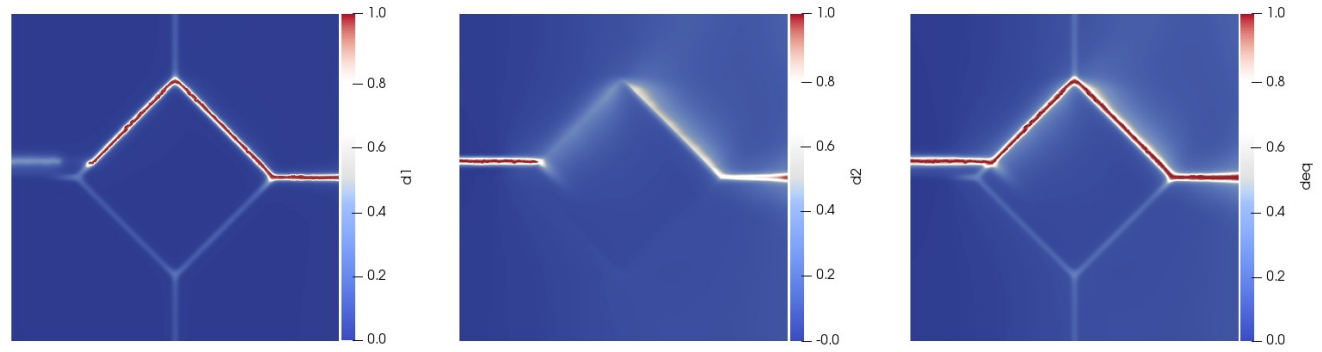

(c)

Figure 8: Crack propagation in 5 grains polycrystal for the three numerical configurations : (a) case 1: $G_{c}^{G B}=0.8 G_{c},(\mathrm{~b})$ case $2: G_{c}^{G B}=0.6 G_{c}$ and (c) case $3: G_{c}^{G B}=0.3 G_{c}$ 


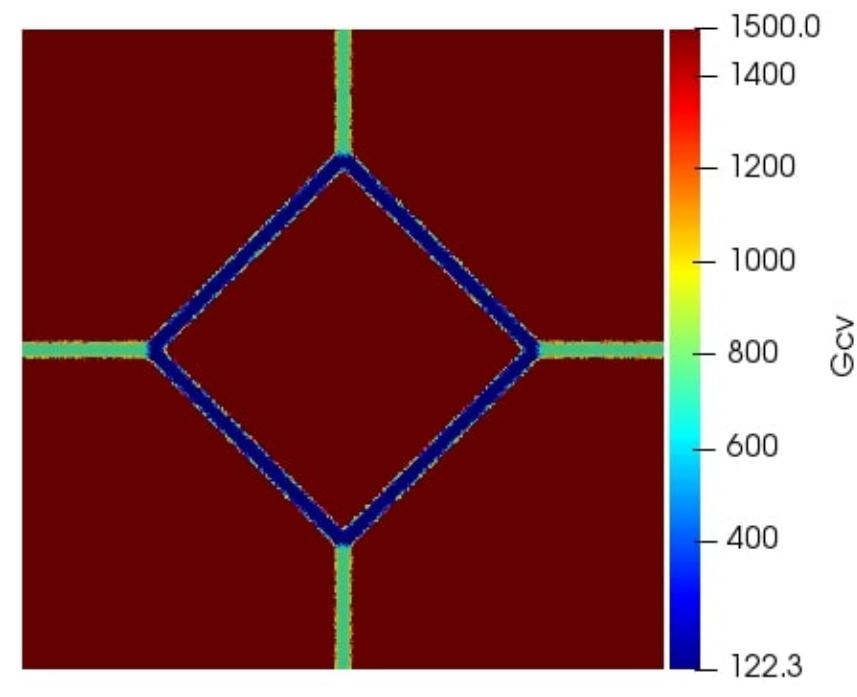

Figure 9: The element-wise field of fracture energy variation as a function of space and grain boundaries misorientation for $G_{c}^{G B}=0.6 G_{c}$

represented in Fig 9. The result of the corresponding intergranular crack propagation is depicted in Fig 10 Contrary to the simulation where misorientation is not accounted for, the crack runs within the grain boundary because $G_{c}^{G B M}$ is much smaller than the previous case in this area comparing to grains fracture energy. A comparison of the result of this simulation with that of the previous example (case 2) highlights the importance of taking grain boundaries misorientation into account in the mechanical properties of grain boundaries.

\subsection{Crack propagation in a polycrystal}

In this last example, we investigate the failure of a more complex polycrystal. A square structure containing 10 grains is generated by the open source software Neper. The mesh consists of 367610 triangular linear elements. The geometric setup as well as the loading are illustrated in Fig. 11a. In the crystallographic axis system, the elastic tensor described in the previous example 3.2 is used.

The fracture energy in the grains is set as in the previous example to $G_{c}=1500 \mathrm{~J} / \mathrm{m}^{2}$. The regularization parameter is set to $l=0.07 \mu \mathrm{m}$ and the grain boundaries thickness is 

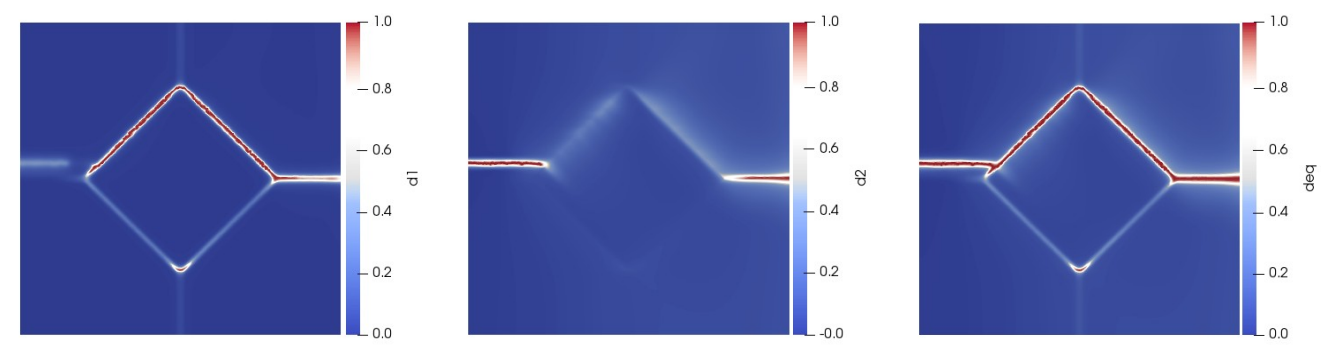

Figure 10: Intergranular failure in the polycrystal for the second case with taking into account grain boundary misorientation.

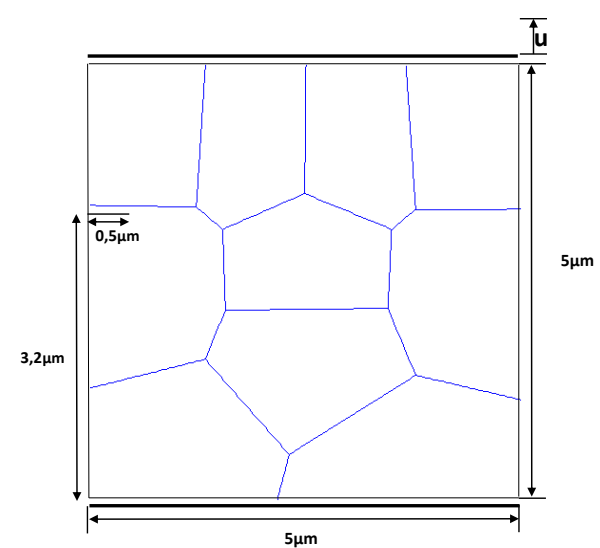

(a)

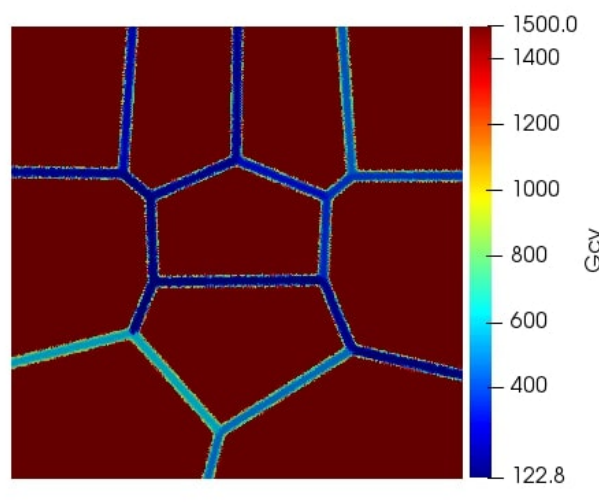

(b)

Figure 11: (a) Geometry, boundary conditions and grain direction for the polycrystalline structure containing 10 grains, (b) the element-wise field of fracture energy variation when it is made dependent on grain boundaries misorientation (case2) 
set to $e_{p}=0.075 \mu \mathrm{m}$. The numerical parameters for fracture energy variation are set to $G_{c}^{B}=10 \times G_{c}$ and $e_{p}^{*}$ is calculated by Eq 33 . To study the effects of fracture energy heterogeneity at the polycrystal scale and between grain boundaries, phase field simulations were performed with different sets of parameters: (1) $G_{c}^{G B}=0.55 G_{c} ;(2) G_{c}^{G B}=0.55 G_{c}$ considering grain boundary misorientation (spacial variation represented in Fig 11). The grain boundaries fracture energy to be applied in the simulations are obtained by the compensation procedure Fig B.2 The fracture energy in the grain boundaries is chosen to promote transgranular fracture in the first case. In the second case, we take into account grain boundary misorientation to promote intergranular fracture.

Two cleavage planes in crystallographic orientations are chosen [1 0$]$ and [0 1] with $\beta=$ 20, corresponding to two damage variables $d_{1}$ and $d_{2}$. The damage variable associated to the grain boundaries is denoted $d_{1}$. The equivalent damage variable $d_{e q}$ is defined by:

$$
d_{e q}=1-\prod_{i}\left(1-d_{i}\right)
$$

The displacement is prescribed along the $y$-direction for the upper edge while the displacement along $x$ are free. The displacement increments are: $\Delta u=0.8 \times 10^{-4} \mathrm{~mm}$ up to $u=2.7 \times 10^{-4} \mathrm{~mm}$ and then $\Delta u=1 \times 10^{-6} \mathrm{~mm}$ up to failure.

Crack propagation results are depicted in Fig 12 for these two configurations. Fig $12 \mathrm{a}$ shows a transgranular fracture, where the crack changes its orientation depending on grain orientation. When grain boundary misorientation is taken into account, intergranular fracture is promoted (see Fig 12b). Another important point is that simulations highlight the capacity of the proposed model to capture initiation and coalescence of additional cracks at grain boundaries. 

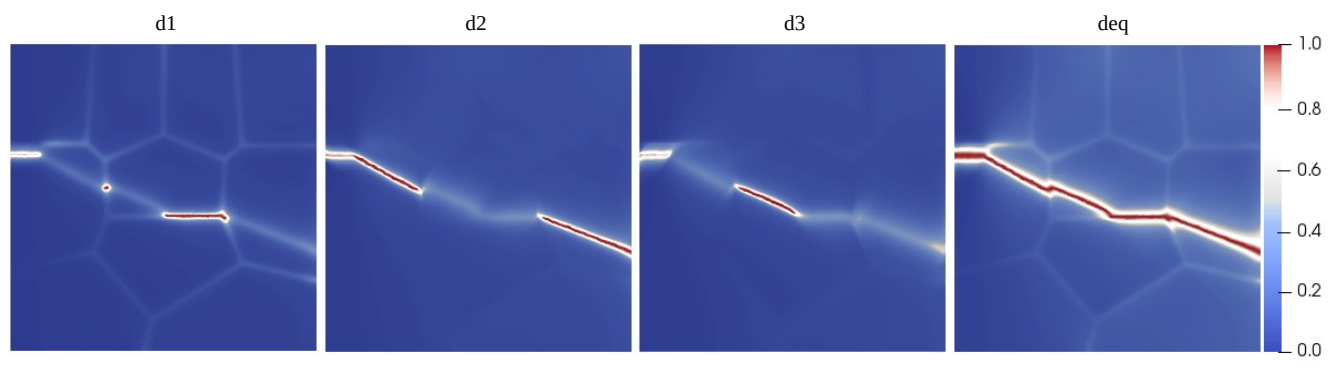

(a)
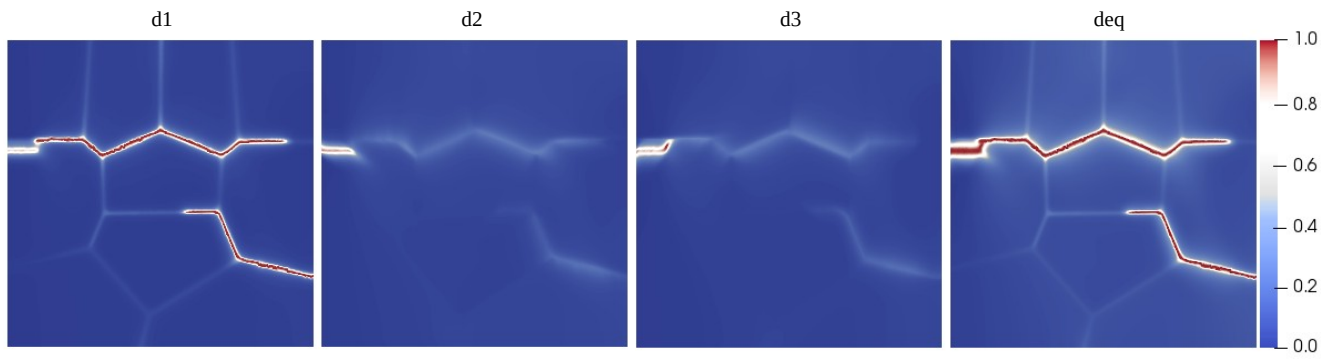

(b)

Figure 12: Crack propagation in a polycrystal with 10 grains for $G_{c}^{G B}=0.55 G_{c}$ without (a) and with misorientation (b). 


\section{Conclusion}

In this paper, is described a unified phase field model to simulate failure mechanisms in polycrystalline materials. The failure along grain boundaries is described by an additional damage variable taking into account the heterogeneous fracture behaviour at the polycrystal scale. This has been made possible thanks to spatial variation of the associated fracture energy. This approach is an alternative to cohesive zone models frequently used in the literature to simulate failure along grain boundaries. Bulk elastic anisotropy and anisotropic surface energy are also considered in the proposed framework. Concerning anisotropic transgranular fracture, many preferential cleavage planes can be considered and a correction was proposed in order to provide more accurate representation of polycrystal physics. It was indeed demonstrated through load-displacement curves analysis that when an anisotropic surface energy is used, the directionality formalism increases the material fracture energy in the preferential direction. To prevent this issue, a numerical correction is proposed on the fracture energy and on the internal length.

The proposed model provides a powerful tool to simulate both intergranular and transgranular fracture and their potential complex competition/interactions. For the additional phase field dedicated to grain boundaries, a numerical compensation was proposed to ensure that crack propagation along grain boundaries is energetically independent from the ratio between the bulk and the grain boundaries fracture energies. This paper also highlighted the importance of the dependence of fracture energy to grain boundary misorientation since it can induce strong modifications of the failure scenarios. The model is also able to deal with crack initiation, coalescence and bifurcation.

Compared to existing modeling strategies using cohesive zone models for the grain boundaries, the proposed model makes use of a thick interface between the grains. This description might be more realistic as interfaces between grains are not perfect interfaces due to the underlying lattice structure of the grains. Further, many particles or impurities localize along grain boundaries what makes a perfect interface of zero-thickness even less representative of their actual geometry. Further, from a mechanical point of view, cohesive zone models ignore the contribution of the tangent stress to the interface. Again, in the case of imperfect interfaces and/or close to junctions like triple points, this 
assumption might be limiting and using a damage model accounting for the contribution of the full stress / strain tensor should be more appropriate.

Future developments might regard structural effects (e.g. accounting for local rotation) of polycrystal microstructure on the interaction between grain boundaries degradation and bulk damage.

\section{Appendix A. Fracture energy variation function}

In the literature, three functions are studied for varying the fracture energy [24]: (i) the Heaviside-like function, (ii) the exponential function and (iii) the Gaussian-like function. The first function leads to convergence problems due to the sharp transition between the bulk and the grain boundary. The second and the third functions provide a smeared transition between the fracture energies, however, they introduce a transition zone that makes difficult controlling the actual grain boundary thickness $e_{p}$. To circumvent these issues, we propose a sinusoidal function Eq. 27 to ensure a continuous transition between fracture energies while approximately preserving the grain boundary thickness. Fig A.1 presents curves allowing for a comparison between the listed functions. The argument of the chosen function Eq27 is set to the power 3 in order to maintain the $G_{c}^{G B}$ value on a larger part of the grain boundary thickness. Then the grain boundary area is described without requiring a numerically expensive mesh. 


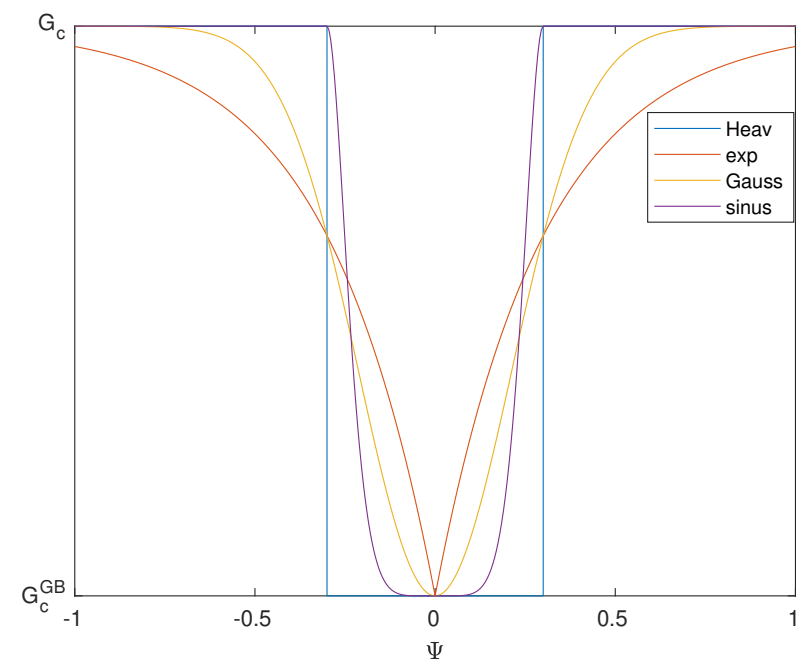

Figure A.1: Fracture energy variation for the different functions: the Heaviside-like function 'Heav', the exponential function 'exp', the Gaussian-like function 'Gauss' and the sinusoidal function 'sinus'.

\section{Appendix B. Numerical compensation for the sinusoidal function}

As it was done for the Heaviside function, exponential function and the Gaussian function 24], we propose here a numerical compensation procedure for the sinusoidal function. The procedure consists in calculating the dissipated energies for different fracture energies in a homogeneous solid with a constant fracture energy $G_{c}$ (reference case : $\mathrm{C} 1)$. Then, for the same set of input parameter assigned to the interface located along the path followed by the crack in the homogeneous case, with a fixed bulk fracture energy, we compute the dissipated energies (interface failure case : C2). The simulations use the geometry and the boundary conditions of the classical benchmark problem shown in Fig 4 The material parameters are set the same as in 3.2 The fracture energy is varying between $0.05 G_{c}$ and $0.95 G_{c}$. The displacement of the top edge is controled by increments: $\Delta u=1 \times 10^{-5} \mathrm{~mm}$ up to $u=3 \times 10^{-3} \mathrm{~mm}$ and then $\Delta u=5 \times 10^{-7} \mathrm{~mm}$ up to failure. Fig B.2 shows comparison of the dissipation as a function of the fracture energy for the reference and the interface failure cases. The compensation consists in determining, for a given grain boundary fracture energy $G_{c}^{G B}$, the corrected $G_{c}^{G B *}$ to assign to the interface in the simulation in order to dissipate the same energy as in the 


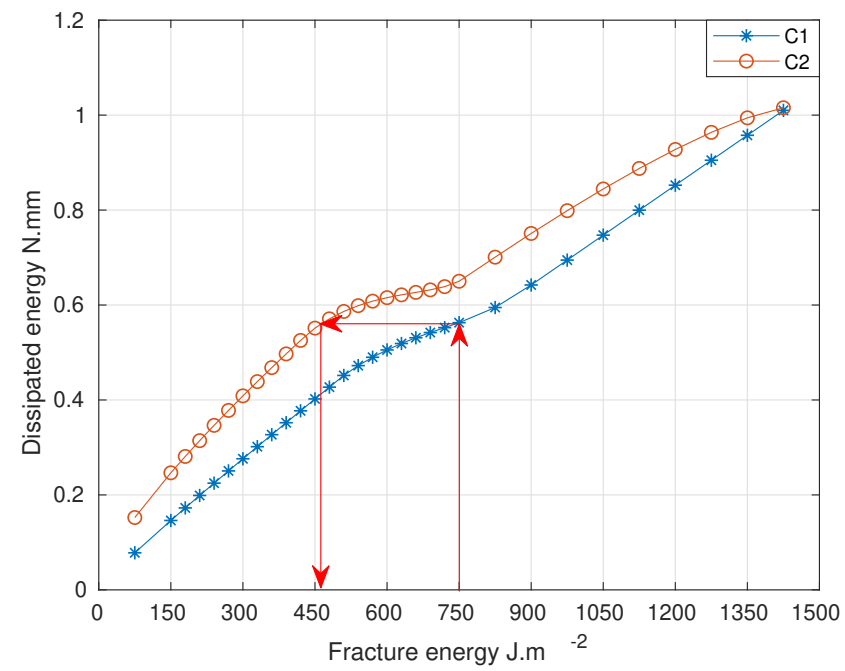

Figure B.2: The graphical compensation of the bulk influence for the sinusoidal function with a fixed ratio $e_{p} / l=1.25$. Red arrows show the compensation procedure for a $G_{c}^{G B}=0.5 G c$.

reference case. A graphical representation is proposed in Fig. B.2. In the proposed example case, for $G_{c}^{G B}=0.5 G c=750 \mathrm{~J} / \mathrm{m}^{2}, G_{c}^{G B *} \approx 467.59 \mathrm{~J} / \mathrm{m}^{2}$. In practice, interpolation in the discrete dataset is used to find the value to assign to $G_{c}^{G B *}$ to get the same energy dissipation as in the ideal case when failure energy is set to though value $G_{c}^{G B}$.

\section{References}

[1] F. Galliano, E. Andrieu, C. Blanc, J.-M. Cloue, D. Connetable, G. Odemer, Effect of trapping and temperature on the hydrogen embrittlement susceptibility of alloy 718 611 370-382. doi: $10.1016 /$ j.msea.2014.06.015

URL https://linkinghub.elsevier.com/retrieve/pii/S0921509314007217

[2] J. Chen, M. Salmeron, T. Devine, Intergranular vs transgranular stress corrosion cracking of cu 30-au 26 (5) 739-742. doi:10.1016/0956-716X(92) 90430-M URL https://linkinghub.elsevier.com/retrieve/pii/0956716X9290430M

[3] M. Paggi, M. Corrado, J. Reinoso, Fracture of solar-grade anisotropic polycrystalline silicon: A combined phase field-cohesive zone model approach 330 123-148. doi:10.1016/j.cma.2017.10.021 URL https://linkinghub.elsevier.com/retrieve/pii/S0045782517306928

[4] L. Zhao, D. Nelias, D. Bardel, A. Maynadier, P. Chaudet, B. Marie, On the fracture of multicrystalline silicon wafer 49 (47) 475601. doi:10.1088/0022-3727/49/47/475601 URL https://iopscience.iop.org/article/10.1088/0022-3727/49/47/475601 
[5] X. Huang, W. Ding, Y. Zhu, C. Yang, Crack propagation simulation of polycrystalline cubic boron nitride abrasive materials based on cohesive element method 138 302-314. doi:10.1016/ j.commatsci.2017.07.007 URL https://linkinghub.elsevier.com/retrieve/pii/S0927025617303580

[6] Y. Ayed, C. Robert, G. Germain, A. Ammar, Orthogonal micro-cutting modeling of the ti17 titanium alloy using the crystal plasticity theory $13743-55$. doi:10.1016/j.finel.2017.08.002 URL https://linkinghub.elsevier.com/retrieve/pii/S0168874X17302093

[7] G. Molnár, A. Gravouil, 2d and 3d abaqus implementation of a robust staggered phase-field solution for modeling brittle fracture 130 27-38. doi:10.1016/j.finel.2017.03.002 URL https://linkinghub.elsevier.com/retrieve/pii/S0168874X16304954

[8] T. T. Nguyen, J. Réthoré, M.-C. Baietto, Phase field modelling of anisotropic crack propagation 65 279-288. doi:10.1016/j.euromechsol.2017.05.002 URL https://linkinghub.elsevier.com/retrieve/pii/S0997753816303321

[9] B. Bourdin, G. Francfort, J.-J. Marigo, Numerical experiments in revisited brittle fracture 48 (4) 797-826. doi:10.1016/S0022-5096(99)00028-9 URL http://linkinghub.elsevier.com/retrieve/pii/S0022509699000289

[10] B. Bourdin, G. A. Francfort, J.-J. Marigo, The variational approach to fracture 91 (1) 5-148. doi: 10.1007/s10659-007-9107-3 URL http://link.springer.com/10.1007/s10659-007-9107-3

[11] C. Miehe, M. Hofacker, F. Welschinger, A phase field model for rate-independent crack propagation: Robust algorithmic implementation based on operator splits 199 (45) 2765-2778. doi:10.1016/j. Cma.2010.04.011 URL https://linkinghub.elsevier.com/retrieve/pii/S0045782510001283

[12] C. Miehe, F. Welschinger, M. Hofacker, Thermodynamically consistent phase-field models of fracture: Variational principles and multi-field FE implementations 83 (10) 1273-1311. doi: 10.1002/nme.2861 URL http://doi.wiley.com/10.1002/nme.2861

[13] G. Francfort, J.-J. Marigo, Revisiting brittle fracture as an energy minimization problem 46 (8) 1319-1342. doi:10.1016/S0022-5096(98)00034-9 URL http://linkinghub.elsevier.com/retrieve/pii/S0022509698000349

[14] B. Li, C. Maurini, Crack kinking in a variational phase-field model of brittle fracture with strongly anisotropic surface energy 125 502-522. doi:10.1016/j.jmps.2019.01.010 URL https://linkinghub.elsevier.com/retrieve/pii/S0022509618308664

[15] L. Chen, B. Li, R. Borst, Adaptive isogeometric analysis for phase-field modeling of anisotropic brittle fracture nme.6457doi:10.1002/nme.6457 URL https://onlinelibrary.wiley.com/doi/abs/10.1002/nme.6457

[16] L. Chen, B. Li, R. de Borst, The use of powell-sabin b-splines in a higher-order phase-field model for crack kinking 67 (1) 127-137. doi:10.1007/s00466-020-01923-0 URL http://link.springer . com/10.1007/s00466-020-01923-0 
[17] B. Yin, M. Kaliske, An anisotropic phase-field model based on the equivalent crack surface energy density at finite strain 369 113202. doi:10.1016/j.cma.2020.113202 URL https://linkinghub.elsevier.com/retrieve/pii/S004578252030387X

[18] T. Nguyen, J. Yvonnet, Q.-Z. Zhu, M. Bornert, C. Chateau, A phase-field method for computational modeling of interfacial damage interacting with crack propagation in realistic microstructures obtained by microtomography 312 567-595. doi:10.1016/j.cma.2015.10.007 URL https://linkinghub.elsevier.com/retrieve/pii/S0045782515003266

[19] T.-T. Nguyen, J. Réthoré, J. Yvonnet, M.-C. Baietto, Multi-phase-field modeling of anisotropic crack propagation for polycrystalline materials 60 (2) 289-314. doi:10.1007/s00466-017-1409-0 URL http://link . springer .com/10.1007/s00466-017-1409-0

[20] M. Paggi, J. Reinoso, Revisiting the problem of a crack impinging on an interface:a modeling framework for the interaction between the phase field approach for brittle fracture and the interface cohesive zone model 321 145-172. doi:10.1016/j.cma.2017.04.004 URL https://linkinghub.elsevier.com/retrieve/pii/S0045782516317066

[21] A. C. Hansen-Dörr, P. Hennig, M. Kästner, K. Weinberg, A numerical analysis of the fracture toughness in phase-field modelling of adhesive fracture 17 (1) 249-250. doi:10.1002/pamm.201710094 URL http://doi .wiley . com/10.1002/pamm. 201710094

[22] A. C. Hansen-Dörr, M. Gude, R. Böhm, M. Kästner, Phase-field modelling of fracture in heterogeneous materials 18 (1). doi:10.1002/pamm.201800082 URL https://onlinelibrary.wiley.com/doi/abs/10.1002/pamm.201800082

[23] A. C. Hansen-Dörr, R. de Borst, P. Hennig, M. Kästner, Phase-field modelling of interface failure in brittle materials 346 25-42. doi:10.1016/j.cma.2018.11.020. URL https://linkinghub.elsevier.com/retrieve/pii/S0045782518305772

[24] A. C. Hansen-Dörr, F. Dammaß, R. de Borst, M. Kästner, Phase-field modeling of crack branching and deflection in heterogeneous media 232 107004. doi:10.1016/j.engfracmech.2020.107004 URL https://linkinghub.elsevier.com/retrieve/pii/S0013794419315474

[25] A. Braides, P. D. o. M. A. Braides, Approximation of Free-Discontinuity Problems, Springer Science \& Business Media, google-Books-ID: 5Ks3FY29MtoC.

[26] M. Ambati, T. Gerasimov, L. De Lorenzis, A review on phase-field models of brittle fracture and a new fast hybrid formulation 55 (2) 383-405. doi:10.1007/s00466-014-1109-y. URL http://link.springer.com/10.1007/s00466-014-1109-y

[27] H. Amor, J.-J. Marigo, C. Maurini, Regularized formulation of the variational brittle fracture with unilateral contact: Numerical experiments 57 (8) 1209-1229. doi:10.1016/j.jmps.2009.04.011 URL https://linkinghub.elsevier.com/retrieve/pii/S0022509609000659

[28] J. Clayton, J. Knap, Phase field modeling of directional fracture in anisotropic polycrystals 98 158-169. doi:10.1016/j.commatsci.2014.11.009 URL https://linkinghub.elsevier .com/retrieve/pii/S0927025614007769

[29] T.-T. Nguyen, J. Bolivar, J. Réthoré, M.-C. Baietto, M. Fregonese, A phase field method for modeling stress corrosion crack propagation in a nickel base alloy 112 65-82. doi:10.1016/j. 
ijsolstr.2017.02.019

URL https://linkinghub.elsevier.com/retrieve/pii/S0020768317300744

[30] D. Mainprice, G. E. Lloyd, M. Casey, Individual orientation measurements in quartz polycrystals: advantages and limitations for texture and petrophysical property determinations 15 (9) 1169-1187. doi:10.1016/0191-8141(93)90162-4

URL https://linkinghub.elsevier.com/retrieve/pii/0191814193901624

[31] T. Luther, C. Könke, Polycrystal models for the analysis of intergranular crack growth in metallic materials 76 (15) 2332-2343. doi:10.1016/j.engfracmech.2009.07.006 URL https://linkinghub.elsevier.com/retrieve/pii/S0013794409002185

[32] Accueil | cast3m. URL http://www-cast3m.cea.fr/

[33] Neper: Polycrystal generation and meshing - neper 4.0.0 documentation URL http://www.neper .info/ 